\title{
Synthesis of catalysts based on magnetic particles $\mathrm{CoFe}_{2} \mathrm{O}_{4}$
}

\author{
Olena Makido, Galyna Khovanets', Oksana Khavunko
}

Department of Physical Chemistry of Fossil Fuels of the Institute of Physical-Organic Chemistry and Coal Chemistry named after L. M. Lytvynenko NAS of Ukraine, Ukraine, Lviv, 79060, Naukova Str., 3a,

E-mail: khovanets_galyna@ukr.net

Abstract - Magnetically sensitive catalysts of the core-shell type based on stabilized particles of cobalt ferrite and copper oxide by the sol-gel method were obtained. A multistage manufacturing technology of the composite $\mathrm{CoFe}_{2} \mathrm{O}_{4} / \mathrm{SiO}_{2} / \mathrm{CuO}$ is proposed. Qualitative and quantitative microanalysis of the obtained composite were carried out and its catalytic action was investigated in the process of the initiated oxidation of cumene. The resulting nanocatalyst is promising for use in the oxidation processes of hydrocarbons.

Keywords - heterogeneous catalysis, sol-gel synthesis, core-shell type catalysts, TEOS, initiated oxidation of cumene.

\section{Introduction}

The creation of catalytic systems based on a magnetic core stabilized by silicon oxide and active centers on the surface of the particles is a topical issue both for chemical synthesis and for ecology and restoration of water resources. Such porous catalysts with magnetic properties (coreshell type) have several advantages: good adsorption capacity, the possibility of magnetic separation and recirculation at room temperature and heat resistance.

\section{Results and discussion}

The developed method for the synthesis of nanostructured magnetically sensitive catalysts of the core-shell type $\mathrm{CoFe}_{2} \mathrm{O}_{4} / \mathrm{SiO}_{2} / \mathrm{CuO}$ includes three stages. At the first stage, the synthesis of cobalt ferrite particles, which form a magnetically sensitive core. The second one is the application of an intermediate layer of silica by sol-gel method. The third stage is the formation of an outer layer of copper oxide, which exhibits catalytic properties.

The obtaining of $\mathrm{CoFe}_{2} \mathrm{O}_{4}$ nanoparticles was carried out by coprecipitation from a solution of iron (III) and cobalt (II) nitrates (at a molar ratio of 2:1) by adding ammonium hydroxide in the presence of surfactant under intense mixing at $80^{\circ} \mathrm{C}$.

The reaction of synthesis can be represented by the following equation: $2 \mathrm{Fe}\left(\mathrm{NO}_{3}\right)_{3}+\mathrm{Co}\left(\mathrm{NO}_{3}\right)_{2}+8 \mathrm{NH}_{4} \mathrm{OH} \rightarrow \mathrm{Co}(\mathrm{OH})_{2}+\mathrm{Fe}(\mathrm{OH})_{3}+8 \mathrm{NH}_{4} \mathrm{NO}_{3}+4 \mathrm{H}_{2} \mathrm{O}$

After evaporation and calcination of the obtained hydroxides at a temperature of $300{ }^{\circ} \mathrm{C}$, porous particles of cobalt ferrite with a developed specific surface and pronounced magnetic properties are formed (Fig. 1).

The application of an intermediate layer of $\mathrm{SiO}_{2}$ is aimed at stabilizing $\mathrm{CoFe}_{2} \mathrm{O}_{4}$ particles and preventing their aggregation. In addition, the presence in the surface layer of the material silanol groups of silicon dioxide enables the formation of chemical bonds with modifiers at the next steps.

The precipitation of $\mathrm{SiO}_{2}$ was carried out by the method of sol-gel

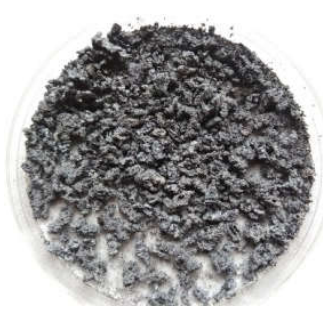

Fig. 1. Photo of $\mathrm{CoFe}_{2} \mathrm{O}_{4}$ particles after annealing synthesis using tetraethoxysilane (TEOS) as a precursor of silicon dioxide. The amount of tetraethoxysilane was calculated from the molar ratio $\mathrm{CoFe}_{2} \mathrm{O}_{4}: \mathrm{SiO}_{2}=2,5: 1$. To particles $\mathrm{CoFe}_{2} \mathrm{O}_{4}$ dispersed in water, tetraethoxysilane was injected in the form of an alcoholic solution. The precipitation was carried out using ammonium hydroxide with vigorous stirring at $40{ }^{\circ} \mathrm{C}$ for 4 hours. The obtained precipitate was washed and left in water in dispersed form. 
To form the catalytic layer, a solution of copper acetate was added to the reactor at the molar ratio $\mathrm{CoFe}_{2} \mathrm{O}_{4}: \mathrm{CuO}=1: 1$. The precipitation of $\mathrm{Cu}(\mathrm{OH})_{2}$ on the surface of the formed $\mathrm{CoFe}_{2} \mathrm{O}_{4} / \mathrm{SiO}_{2}$ particles was carried out by adding an equimolar amount of $\mathrm{NaOH}$. After washing to $\mathrm{pH}=7$ and drying at $100{ }^{\circ} \mathrm{C}$ for 24 hours, the obtained precipitate was calcined at $300{ }^{\circ} \mathrm{C}$ for 1 hour. With this technology, the formation of copper oxide on the surface of the particles, which is bonded to the particle by chemical bond.

The study of the morphology of the obtained composites showed that the particles of cobalt ferrite have a finely dispersed structure of micron sizes (Fig. 2a). The analysis of the obtained spectrums indicates that, in addition to cobalt ferrite, part of the atoms of the ferrum exists in the form of the mineral of maghemite $\gamma-\mathrm{Fe}_{2} \mathrm{O}_{3}$ (Fig. 2b). When applied to the surface of cobalt ferrite particles of a dense layer of $\mathrm{SiO}_{2}$ (Fig. 2c) and a layer of copper oxide (Fig. 2d), an increase in the size of particles with the preservation of the developed surface is observed.

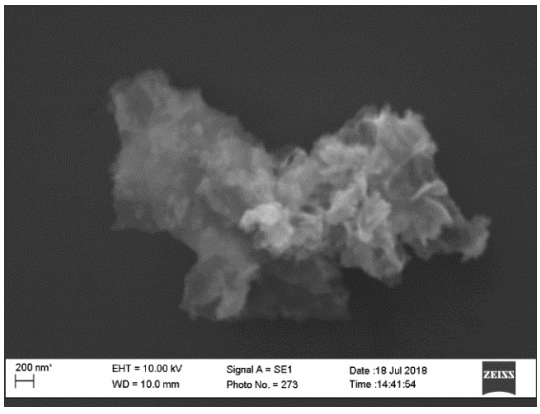

a)

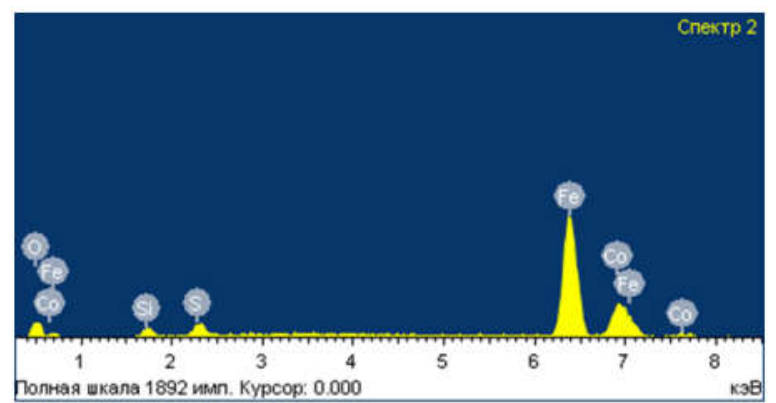

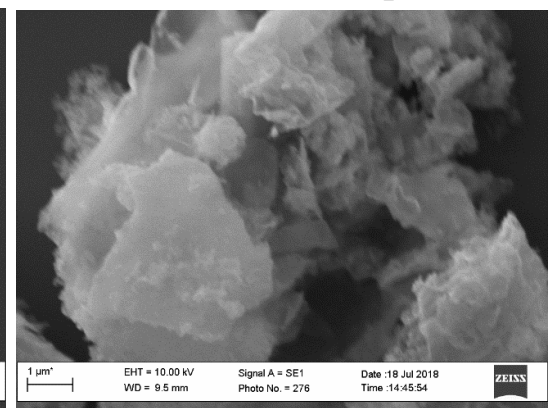

b)

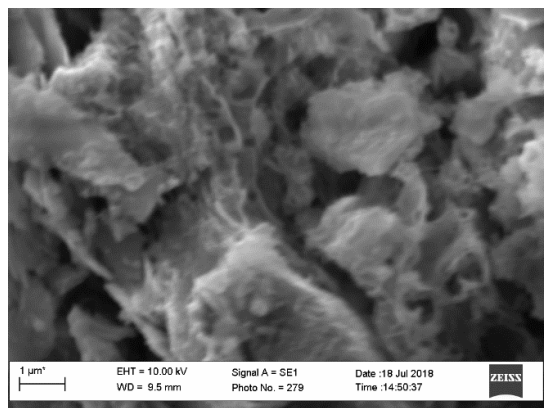

c)

\begin{tabular}{l|ll} 
Элемент & Весовой \% & Атомный\% \\
O K & 7.27 & 21.00 \\
$\mathrm{Si} \mathrm{K}$ & 2.10 & 3.46 \\
$\mathrm{~S} \mathrm{~K}$ & 2.19 & 3.16 \\
$\mathrm{Fe} \mathrm{K}$ & 69.09 & 57.20 \\
Со K & 19.35 & 15.18 \\
& & \\
Итоги & 100.00 &
\end{tabular}

d)

Fig. 2. SEM photographs of samples of synthesized composites: $\mathrm{CoFe}_{2} \mathrm{O}_{4}$ (a), $\mathrm{CoFe}_{2} \mathrm{O}_{4} / \mathrm{SiO}_{2}$ (b), $\mathrm{CoFe}_{2} \mathrm{O}_{4} / \mathrm{SiO}_{2} / \mathrm{CuO}$ (c) and spectrogram of the sample composition $\mathrm{CoFe}_{2} \mathrm{O}_{4}$ (d)

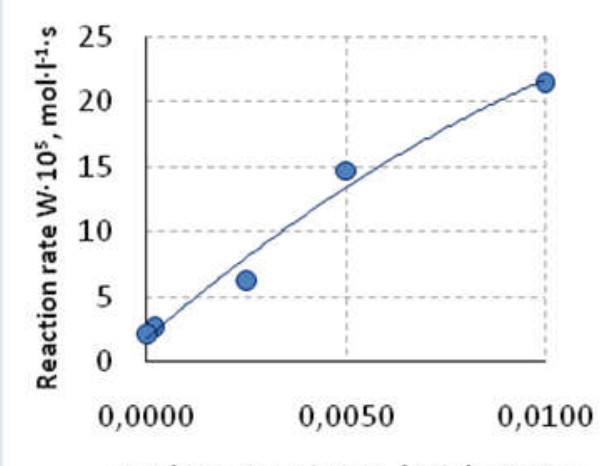

Catalyst mass $\mathrm{CoFe}_{2} \mathrm{O}_{4} / \mathrm{SiO}_{2} / \mathrm{CuO}$ m, g

Fig. 3. Dependence of the reaction rate of initiated cumene oxidation

on the amount of catalyst

$$
\mathrm{CoFe}_{2} \mathrm{O}_{4} / \mathrm{SiO}_{2} / \mathrm{CuO}
$$

The catalytic action of the obtained composite was determined in the process of initiated oxidation of cumene by molecular oxygen. The use of the composite $\mathrm{CoFe}_{2} \mathrm{O}_{4} / \mathrm{SiO}_{2} / \mathrm{CuO}$ as a catalyst in the process of cumene oxidation allows to increase in the process rate by an order, depending on the amount of the used catalyst (Fig. 3). In addition, the obtained catalyst demonstrated the ability to work for several cycles without regeneration and without loss of catalytic activity.

\section{Conclusion}

The research results showed that the composite $\mathrm{CoFe}_{2} \mathrm{O}_{4} / \mathrm{SiO}_{2} / \mathrm{CuO}$, made a according to the proposed technology, can serve as a catalyst for the process of initiated cumene oxidation. 